\title{
A stemness-based eleven-gene signature correlates with the clinical outcome of hepatocellular carcinoma
}

Liang Hong ${ }^{1}$, Yu Zhou' ${ }^{1}$ Xiangbang Xie ${ }^{2}$, Wanrui Wu ${ }^{2}$, Changsheng Shi ${ }^{2}$, Heping Lin ${ }^{2,3^{*}}$ and Zhenjing Shi ${ }^{2^{*}}$

\begin{abstract}
Background: Cumulative evidences have been implicated cancer stem cells in the tumor environment of hepatocellular carcinoma (HCC) cells, whereas the biological functions and prognostic significance of stemness related genes (SRGs) in HCC is still unclear.

Methods: Molecular subtypes were identified by cumulative distribution function (CDF) clustering on 207 prognostic SRGs. The overall survival (OS) predictive gene signature was developed, internally and externally validated based on HCC datasets including The Cancer Genome Atlas (TCGA), GEO and ICGC datasets. Hub genes were identified in molecular subtypes by protein-protein interaction (PPI) network analysis, and then enrolled for determination of prognostic genes. Univariate, LASSO and multivariate Cox regression analyses were performed to assess prognostic genes and construct the prognostic gene signature. Time-dependent receiver operating characteristic (ROC) curve, Kaplan-Meier curve and nomogram were used to assess the performance of the gene signature.
\end{abstract}

Results: We identified four molecular subtypes, among which the C2 subtype showed the highest SRGs expression levels and proportions of immune cells, whereas the worst OS; the C1 subtype showed the lowest SRGs expression levels and was associated with most favorable OS. Next, we identified 11 prognostic genes (CDX2, PON1, ADH4, RBP2, LCAT, GAL, LPA, CYP19A1, GAST, SST and UGT1A8) and then constructed a prognostic 11-gene module and validated its robustness in all three datasets. Moreover, by univariate and multivariate Cox regression, we confirmed the independent prognostic ability of the 11-gene module for patients with HCC. In addition, calibration analysis plots indicated the excellent predictive performance of the prognostic nomogram constructed based on the 11gene signature.

Conclusions: Findings in the present study shed new light on the role of stemness related genes within HCC, and the established 11-SRG signature can be utilized as a novel prognostic marker for survival prognostication in patients with HCC.

Keywords: Stemness, Hepatocellular carcinoma, Gene signature, Molecular subtype, Prognosis

\footnotetext{
*Correspondence: 13857793035@163.com; szi258@126.com

2Department of Interventional, The Third Affiliated Hospital of Wenzhou Medical University, Ruian, Zhejiang 325200, People's Republic of China

Full list of author information is available at the end of the article
}

(c) The Author(s). 2021 Open Access This article is licensed under a Creative Commons Attribution 4.0 International License, which permits use, sharing, adaptation, distribution and reproduction in any medium or format, as long as you give appropriate credit to the original author(s) and the source, provide a link to the Creative Commons licence, and indicate if changes were made. The images or other third party material in this article are included in the article's Creative Commons licence, unless indicated otherwise in a credit line to the material. If material is not included in the article's Creative Commons licence and your intended use is not permitted by statutory regulation or exceeds the permitted use, you will need to obtain permission directly from the copyright holder. To view a copy of this licence, visit http://creativecommons.org/licenses/by/4.0/ The Creative Commons Public Domain Dedication waiver (http://creativecommons.org/publicdomain/zero/1.0/) applies to the data made available in this article, unless otherwise stated in a credit line to the data. 


\section{Background}

Globally, liver cancer is the fourth most lethal cancer worldwide [1]. Hepatocellular carcinoma (HCC) is rank as the major histological subtype (approx. 70-85\% cases) of total liver cancer cases. The prognosis of advanced $\mathrm{HCC}$ is still not satisfactory and treatment options are limited [2]. Integrative studies combining transcriptome and genomic analysis have confirmed that $\mathrm{HCC}$ has much heterogeneous at the histo-molecular level and clinical outcomes, and the molecular diversity of HCC is tightly associated with different aetiologies and distinct mechanisms of hepato-carcinogenesis [2]. Give that only individually tailored molecular profiles and biomarkers could escape the patients from undergoing a potentially more harmful, aggressive chemical therapy or even leave them untreated, we should illustrate the natural history of HCC in individual patients by clearly understood their personal molecular characteristics. Therefore, there is an increasing interest in the molecular characterization of HCC allowing prognosticate overall patient survival.

The biological similarity of cancer cells and stem cells has been well documented, and abnormal stem cells is supposed to play an important role in HCC progression [3]. Although several highly conserved pathways including Notch, Hedgehog, Hypoxia and Wnt signaling pathways are pivotal for maintaining self-renewal in cancer stem cells (CSCs) and thus involved in tumorigenesis and cancer development [4], almost nothing is known about the precise role and underlying mechanism of stemness related genes (SRGs) and their gene expression profiles in primary HCC, not yet anything known related to the prognostic distinctions of HCC.

Here we aimed at explore new prognostic signature in patients with HCC using Cox and the least absolute shrinkage and selection operator (LASSO) regression models to analyze the expression profile of stemnessrelated genes (SRGs) using multiple online HCC dataset. Based on SRG expression data from public databases in The Cancer Genome Atlas (TCGA), we constructed molecular subtypes with distinct different immune characteristics and clinical outcomes. Then, we developed a 11-gene signature for assessing the prognosis of patients with $\mathrm{HCC}$, and further validated it in TCGA, Gene Expression Omnibus (GEO) and International Cancer Genome Consortium (ICGC) HCC datasets. This module was closely related to patients' prognosis and could apply as an independent pathological predictor.

\section{Methods}

\section{Patients and datasets}

The clinical information and RNA-seq data of $342 \mathrm{HCC}$ samples were download from TCGA database (http:// www.cancer.gov/about-nci/organization/ccg/research/ structural-genomics/TCGA). A dataset which contains gene expression profiles of HCC samples in GEO database, GSE15654 (contains 216 HCC samples), was downloaded from NCBI (http://www.ncbi.nlm.nih.gov/ geo/). The RNA-seq data and clinical information of 212 HCC samples in the ICGC HCC cohort was download from ICGC Data Portal (https://icgcportal.genomics.cn/). The clinicopathological characteristics of patients from these three datasets after preprocessing are summarized in Table 1. For TCGA HCC dataset, $50 \%$ of them was randomly divided into training cohort $(n=171)$, and the rest 171 cases and the entire dataset were selected as internal validation cohorts. GSE15654 and ICGC HCC datasets were used as external validation cohorts. Patient informed consent was existing in these three public datasets, and this study was approved by the Institutional Review Boards of the Third Affiliated Hospital of Wenzhou Medical University.

\section{Identification of molecular subtypes based on SRGs}

A total of 456 genes related to stemness from 30 stemness-related gene sets (Supplementary Table S1) were collected from the Molecular Signature Database v7.0 (MSigDB). Among them, 48 genes not offered in TCGA HCC dataset or with FPKM $=0$ in more than half of the samples were excluded. Finally, 408 genes were enrolled for subsequent analysis. Prognostic genes were detected by univariate Cox regression survival analysis using the $\mathrm{R}$ package survival coxph function, and $\log$ rank $P<0.05$ was selected as the threshold. The molecular subtypes were identified based on these prognostic genes using cumulative distribution function (CDF) method, and the optimal number of subtypes were determined according to the CDF Delta area.

\section{Identification of hub genes by protein-protein interaction (PPI) analysis}

Since protein-protein interaction (PPI) analysis can help identify hub genes with core functions, PPI among genes in the identified key modules was further explored. The Search Tool for the Retrieval of Interacting Genes (STRI NG) is a well-known database containing comprehensive PPI information (version 11.0, https://string-db.org/). The PPI network of these genes was thus mapped to the STRING assembly and then visualized by the Cytoscape software. Important nodes in the network was identified by the Cytoscape plugin cytoHubba [5]. The topological analysis method Degree and the centrality analysis methods Closeness and Betweenness were used separately to identify the hub nodes in the PPI network. Among the top 10 hub nodes identified by each method, only genes with consistent high Degree, Closeness, and Betweenness values (larger than the median value) were selected as hub genes. 
Table 1 Clinical and pathologic characteristics of patients in the pre-processed TCGA and GEO HCC datasets

\begin{tabular}{|c|c|c|c|c|c|c|}
\hline Characteristic & & $\begin{array}{l}\text { Training Set } \\
(n=171)\end{array}$ & $\begin{array}{l}\text { Validation Set } \\
(n=171)\end{array}$ & $P$ value* & $\begin{array}{l}\text { ICGC HCC dataset } \\
(n=212)\end{array}$ & $\begin{array}{l}\text { GSE15654 dataset } \\
(n=216)\end{array}$ \\
\hline \multirow[t]{2}{*}{ Age (years) } & $\leq 60$ & 81 & 84 & 0.828 & 43 & - \\
\hline & $>60$ & 90 & 87 & & 169 & - \\
\hline \multirow[t]{2}{*}{ Survival Status } & Living & 109 & 110 & 1 & 176 & 150 \\
\hline & Dead & 62 & 61 & & 36 & 66 \\
\hline \multirow[t]{2}{*}{ Sex } & Female & 55 & 54 & 1 & 50 & - \\
\hline & Male & 116 & 117 & & 162 & - \\
\hline \multirow[t]{4}{*}{ Grade } & G1 & 24 & 29 & 0.513 & - & - \\
\hline & G2 & 82 & 79 & & - & - \\
\hline & G3 & 59 & 52 & & - & - \\
\hline & G4 & 4 & 8 & & - & - \\
\hline \multirow[t]{4}{*}{ Pathologic T stage } & $\mathrm{T} 1$ & 79 & 89 & 0.028 & - & - \\
\hline & $\mathrm{T} 2$ & 54 & 30 & & - & - \\
\hline & T3 & 43 & 41 & & - & - \\
\hline & T4 & 4 & 9 & & - & - \\
\hline \multirow[t]{2}{*}{ Pathologic N stage } & No & 120 & 119 & 0.934 & - & - \\
\hline & $\mathrm{N} 1 / \mathrm{Nx}$ & 50 & 52 & & - & - \\
\hline \multirow[t]{2}{*}{ Pathologic M stage } & MO & 128 & 116 & 0.188 & - & - \\
\hline & $\mathrm{M} 1 / \mathrm{Mx}$ & 43 & 55 & & - & - \\
\hline \multirow[t]{4}{*}{ Tumor Stage } & Stage I & 78 & 83 & 0.011 & 33 & - \\
\hline & Stage ॥ & 50 & 27 & & 102 & - \\
\hline & Stage III & 34 & 46 & & 61 & - \\
\hline & Stage IV & 1 & 2 & & 16 & - \\
\hline
\end{tabular}

*Chi-Squared test. ICGC, International Cancer Genome Consortium

\section{Construction of stemness-related prognostic gene signature}

Co-expression genes in the training set were detected by univariate Cox regression survival analysis, and $\log$ rank $p<0.01$ was selected as the threshold. To narrow the gene range and maximize the accuracy, LASSO Cox regression analysis [6], a method screening signatures with generally effective prognostication performance by performing automatic feature selection, was performed by using the glmnet package of $\mathrm{R}$ to identify the prognostic gene. Optimal genes were evaluated by 10 -fold cross validation. Risk score for each patient of the training set was calculated with the linear combinational of the signature gene expression weighted by their regression coefficients. Risk score $=\left(\operatorname{expr}_{\text {gene1 }} \mathrm{x}\right.$ coefficient gene1 $)+\left(\operatorname{expr}_{\text {gene2 }} \times\right.$ coefficient $\left._{\text {gene } 2}\right)+\ldots+\left(\operatorname{expr}_{\text {genen }} \quad \mathrm{x}\right.$ coefficient genen $)$. Receiver operating characteristics (ROC) curves, carried out by using the $\mathrm{R}$ package timeROC, was used to analyze the risk score of each sample, and samples were set as high-risk group or low-risk group by set the threshold as 0 .

\section{Bioinformatic analysis}

The enumeration of six tumor-infiltration immune cells (B cell, $\mathrm{CD}^{+} \mathrm{T}$ cell, $\mathrm{CD} 8^{+} \mathrm{T}$ cell, neutrophil, macrophage, neutrophils and dendritic cell) was estimated using the "Tumor Immune Estimation Resource" (TIME R, https://cistrome.shinyapps.io/timer/) tool [7]. Singlesample Gene set enrichment analysis (ssGSEA) was applied for identifying relationship between the risk scores of different samples and biological functions using the $\mathrm{R}$ package GSVA. The classical gene sets of Kyoto Encyclopedia of Genes and Genomes (KEGG) pathways (c2. cp. kegg. v11.0.symbols) were considered to decipher the phenotype. For each analytical pathway, the enrichment score (ES) and the significance of ES were calculated, and the normalized enrichment score (NES) and false discovery rate (FDR) were further calculated to examine functional enrichment results. A FDR cutoff value of 0.05 was considered in this test.

\section{Statistical analysis}

DESeq2 was used to calculate differentially expressed genes (DEGs) among each cluster $(\mathrm{FDR}<0.05$ and 
$|\log 2 \mathrm{FC}|>1)$. Kaplan-Meier curves were applied to assess the difference on OS between different groups. Multivariate Cox regression analyses were performed to assess the independent prognostic factors. Decision curve analysis (DCA), which can evaluate predictive models from the perspective of clinical consequences [8], was performed in the entire cohort to test the clinical usefulness of the nomogram in comparison with the gene signature and clinicopathological parameters. All statistical analyses were using R 3.6.0 (https://mirrors. tuna.tsinghua.edu.cn/CRAN/) with default software parameters. $P$ value $<0.05$ was considered significant statistically.

\section{Result}

\section{Identification of molecular subtypes in HCC}

By univariate Cox regression survival analysis, 207 stemness related genes (SRGs) were identified correlated with the overall survival (OS) of patients with HCC in the TCGA dataset (Supplementary Table S2). Consensus unsupervised clustering of 342 samples from HCC patients, we found that 4 clusters had lower values of ambiguously clustered pairs (PAC), which reflected the nearperfect stability of the samples under the correct $\mathrm{K}$ value distribution (Fig. 1a-b). The relative change of the area under the CDF curve reveals a nearly perfect stable distribution of the samples starting from 4 clusters (Fig. 1c). Principal component analysis (PCA) and consensus heatmaps also showed a relatively stable distribution samples in the 4 clusters (Fig. 1d-e). After evaluating the relative changes in the area under the CDF curve, PAC value, PCA and consensus heatmaps, we chose a fourcluster solution. Thus, four molecular subtypes (Cluster $1[n=82]$, Cluster $2[n=54]$, Cluster $3[n=105])$ and Cluster $4[n=101])$ were constructed based on these 207 prognostic genes (Fig. 1a-e).

Gene expression profile and the distribution of clinicopathological parameters in each subtype was showed in Fig. 1e. However, the molecular subtype had no correlation with any clinicopathological features of patients with HCC (Supplementary Figure 1). Kaplan-Meier method with log-rank tests were applied to explore the difference of prognosis among the four molecular subtypes in HCC. Compared with Cluster 1 and Cluster 3, patients in Cluster 2 and Cluster 4 showed worse overall survival (OS) time (Fig. 1f). In addition, the differences on immune characteristics among the three subtypes were analyzed. Cluster 2 showed the highest proportions of immune cell infiltration than the other three subtypes (Fig. 1g).

Selection of hub genes by intersection and PPI analyses Genes differentially expressed among each molecular subtype were calculated (Table 2). There are 286 differentially expressed genes (DEGs, 272 up-regulated and 14 down-regulated, Supplementary Figure 2A) in Cluster 1 compared with the other three subtypes, 727 DEGs (294 up-regulated and 433 down-regulated, Supplementary Figure 2B) between Cluster 2 and other three subtypes, 276 DEGs (264 up-regulated and 12 down-regulated, Supplementary Figure 2C) between Cluster 3 and other three subtypes, and 56 DEGs (31 up-regulated and 25 down-regulated, Supplementary Figure 2D) between Cluster 4 and other three subtypes. The up-regulated and down-regulated DEGs were depicted on Venn diagram (Supplementary Figure 2E-F).

Considering that gene interaction networks helps to uncover key genes participate in liver cancer progression, we mapped the expression of the 1345 DEGs to STRING database to construct PPI network. A total of 1216 PPI pairs with a score higher than 0.7 were matched (Fig. 2a, Supplementary Table S3). Among which, the top 490 hub genes identified by the Degree (Fig. 2b), Closeness (Fig. 2c), and Betweenness (Fig. 2d) methods were largely consistent (Supplementary Table S4). The topological properties of the PPI network were also evaluated and the distributions of degree, closeness, and betweenness were shown in Fig. 2e-g. A total of 130 genes that satisfied high degree, closeness, and betweenness scores were selected out as hub genes for further analysis (Supplementary Table S5). These hub DEGs were assumed to be strongly correlated with the development of $\mathrm{HCC}$, and were enrolled for subsequently identification of prognostic gene.

\section{Construction of prognosis risk model based on differential co-expression genes}

The clinical information of HCC patients in the TCGA training $(n=171), T C G A$ testing $(n=171)$, and two external validation sets used for model construction and evaluation was listed in Table 1 . To identify novel prognostic marker for patients with $\mathrm{HCC}$, we applied univariate Cox proportional hazard regression and dimensional-reduction analysis by performing LASSO regression analysis to these 130 hub DEGs in the training set. And then 11 genes (including CDX2, PON1, ADH4, RBP2, LCAT, GAL, LPA, CYP19A1, GAST, SST and UGT1A8) significantly correlated to OS $(P<0.01$, Table 3$)$ were confirmed with 10 fold cross-validation and the minimized error rate $\lambda=$ 0.034 (Fig. 3a-b). Among them, CDX2, RBP2, GAL, CYP19A1, GAST, SST and UGT1A8 showed significant negative correlation with OS, while the other four gens, PON1, ADH4, LCAT and LPA, were positive correlated to OS. The final 11-gene signature was calculated using Multivariate Cox survival analysis (Table 3), and a genebased prognostic model was established to evaluate the survival risk of each patient, the formula of the gene signature is as follows: RiskScore $=0.079 * \exp ^{\operatorname{CDX} 2}-0.203 *$ 


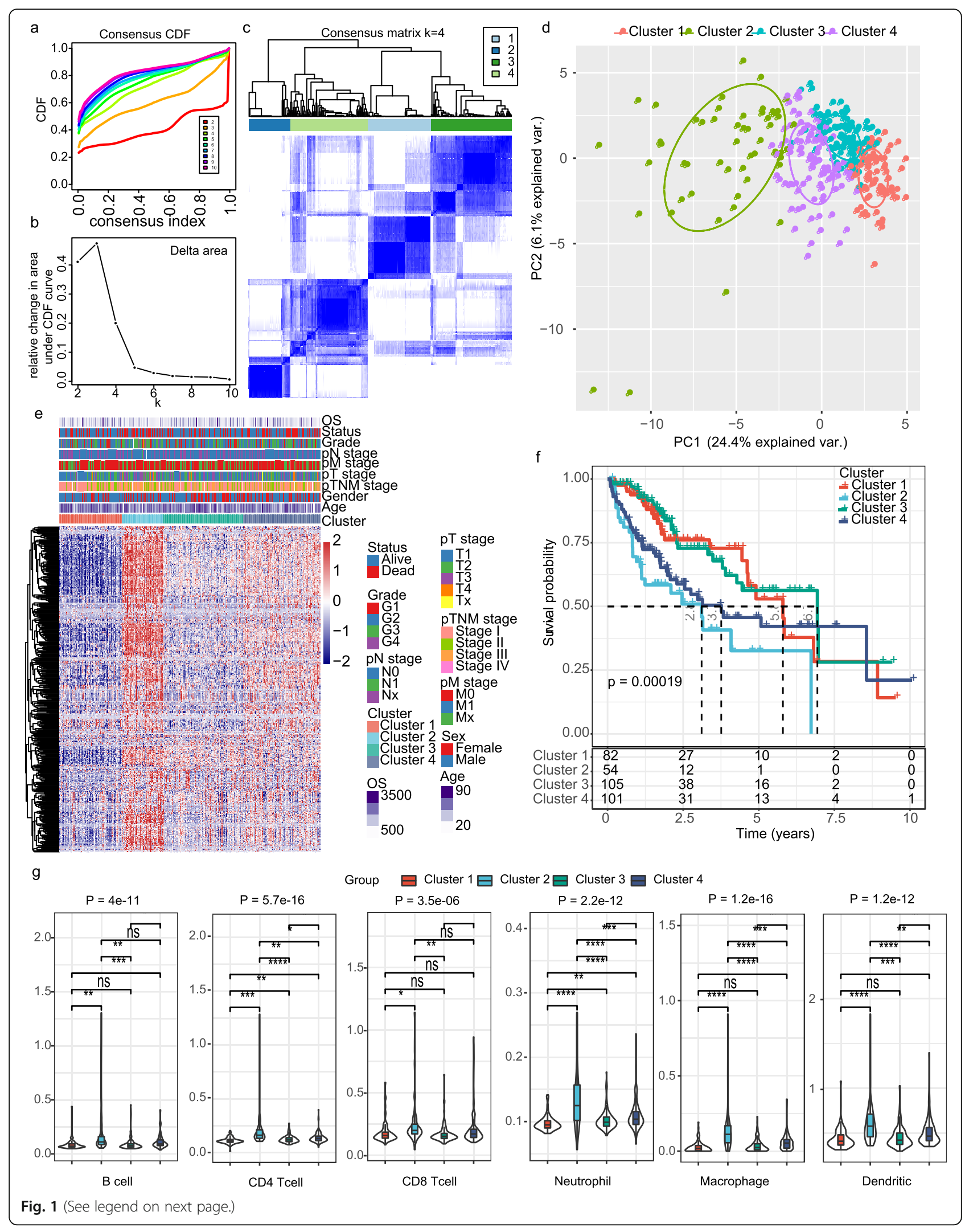


(See figure on previous page.)

Fig. 1 Identification of molecular subtypes in HCC. a cumulative distribution function (CDF) curve of $\mathrm{K}=2-10$; $\mathbf{b}$ The relative change in area under the CDF curve of $K=2-10$; $\mathbf{c}$ A relative stable partition of the samples is found at $K=4$. $\mathbf{d} P C$ analysis for $K=4$ are shown; e Heat map of the expression profile of 408 SRGs and the distribution of clinicopathological parameters in all four subtypes; $\mathbf{f}$ Kaplan-Meier curves with log rank analysis showed the overall survival (OS) curve of the four subtypes; $\mathbf{g}$ The proportions of B cell, CD4 ${ }^{+} T$ cell, $C D 8^{+} T$ cell, Neutrophil, Macrophage, and Dendritic cell (DC) in the three subtypes

$\exp ^{\mathrm{PON} 1}-0.021 * \exp ^{\mathrm{ADH} 4}+0.094 * \exp ^{\mathrm{RBP} 2}+0.297 *$ $\exp ^{\mathrm{LCAT}}+0.177 * \exp ^{\mathrm{GAL}}+0.037 * \exp ^{\mathrm{LPA}}+0.102 *$ $\exp ^{\text {CYP19A1 }}+0.048 * \exp ^{\text {GAST }}+0.102 * \exp ^{\text {SST }}+0.087 *$ $\exp ^{\text {UGT1A8. }}$

Based on the risk score formula and the cut-off value of normalized risk score $(Z$-score $=0)$, patients were divided into high-risk or low-risk group (Fig. 3c). And a heatmap shown the expression profile of the eight genes illustrated that as the risk score of patients increased, the expression of prognosis-risky genes (CDX2, RBP2, GAL, CYP19A1, GAST, SST and UGT1A8) were distinctly upregulated; in contrast, the expression of prognosis-protective gene (PON1, ADH4, LCAT and LPA) were downregulated. ROC curve showed that the accuracy of the 11-gene signature for one-year, three-year and five-year survival were greater than 0.70 (Fig. 3c). Finally, we divided the samples into the high-risk group $(n=79)$ when their Zscore-based Riskscore greater than zero, and the others as the low-risk group $(n=92)$. KaplanMeier curve analysis revealed that high-Riskscore confers significantly shorter OS time $(\mathrm{HR}=2.27,95 \% \mathrm{CI}$ $1.78-2.89 ; P<0.001$; Fig. $3 \mathrm{~d})$. To analyze the clinicopathological correlation of the RiskScore, we obtained vascular invasion and tumor differentiation information from the TCGA dataset, and compare the difference between high- and low-risk groups. For vascular invasion status, although there was no significant difference, sample with vascular invasion has a higher proportion of highrisk patients (Supplementary Figure 3A). In addition, there are significant differences between high- and low-risk patients on tumor differentiation (Supplementary Figure 3B). G1 group has the highest proportion of low-risk patients, and G4 group has the highest proportion of highrisk patients, which suggests that the RiskScore is significantly correlated with tumor differentiation.

Table 2 DEGs in each Cluster

\begin{tabular}{lllll}
\hline & Cluster 1 & Cluster 2 & Cluster 3 & Cluster 4 \\
\hline Upregulated DEGs & 272 & 294 & 264 & 31 \\
Downregulated DEGs & 14 & 433 & 12 & 25 \\
All DEGs & 286 & 727 & 276 & 56 \\
\hline
\end{tabular}

DEGs differentially expressed genes

\section{Validation of the 11-gene signature in the internal dataset and two external HCC datasets}

The TCGA testing dataset $(n=171)$ and the entire TCGA HCC dataset $(n=342)$ were used for internal validation, patients in both two sets were calculated using the same coefficients. Patients were sub-grouped using the same cut-off value as the training set. The corresponding ROC curve and Kaplan-Meier survival curves for the TCGA testing set and the entire TCGA dataset showed that the AUCs of the signature remained high and the high-risk groups had consistently shorter OS periods than the low-risk groups (Fig. 4).

Subsequently, the prognostication efficiency of our 11gene signature was also calculated in the GSE15654 and ICGC HCC cohort. The results showed that the association between the gene expression and risk score was consistent with that in the training and internal validation sets (Fig. 5). In the GSE15654 dataset, The ROC curve revealed that the AUCs of the prognostic 11-gene signature for 1-year, 3-year and 5-year survival were $0.71,0.58$ and 0.62 , respectively (Fig. 5a). As expected, patients in the GSE15654 dataset with high risk-scores had shorter OS than those with low risk scores (HR = 1.44, 95\%CI 1.13-1.84; $P=0.024$; Fig. 5b). In the ICGC HCC cohort, the ROC curve showed that the AUCs of the 11-gene signature for one-year, three-year and fiveyear survival were consistently greater than 0.70 (Fig. 5c). As expected, patients in the ICGC HCC cohort with high risk-scores had shorter OS than those with low risk scores $(\mathrm{HR}=1.71,95 \% \mathrm{CI} 1.31-2.24 ; P=0.011$; Fig. 5d). Therefore, the 11-gene signature displayed solid effective prognostic classification performance in the two external validation sets.

\section{Univariate and multivariate cox regression analyses of the 11-gene signature}

To identify whether the 11-gene signature is an independent prognostic predictor in clinical application, we applied univariate and multivariate Cox regression analysis to systematically evaluate the prognosis of patients in the entire TCGA and ICGC HCC dataset. In the entire TCGA HCC dataset, univariate analysis of survival revealed that the 11 -gene signature $(P<0.001)$, pT stage $(P<0.001), \mathrm{pN}$ stage $(P<0.001), \mathrm{pM}$ stage $(P<0.001)$ and pTNM stage $(P<0.001)$ were prognostic indicators of OS (Fig. 6a). However, multivariate Cox regression analysis showed that only 11 -gene signature $(P<0.001)$ 
a
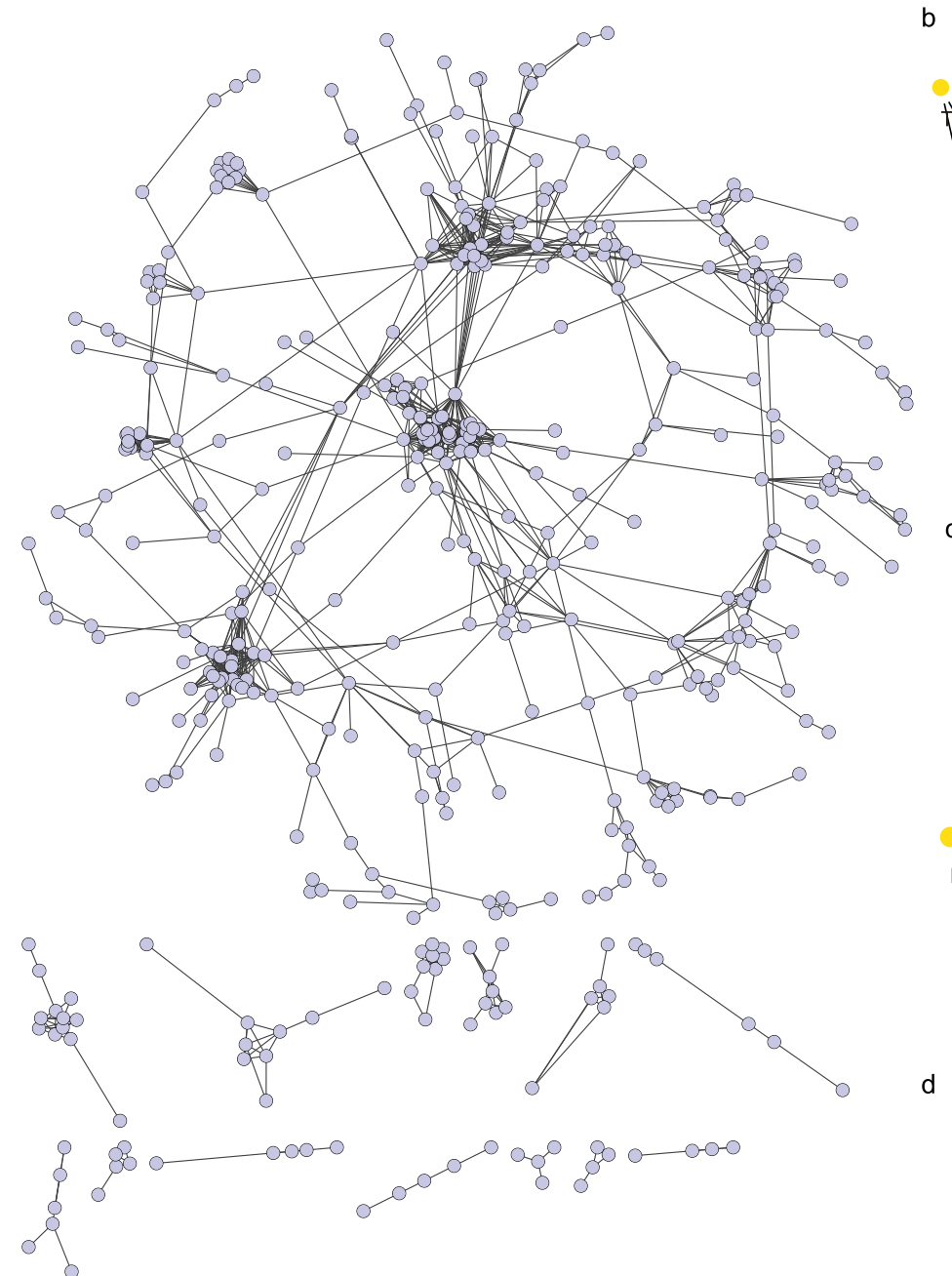

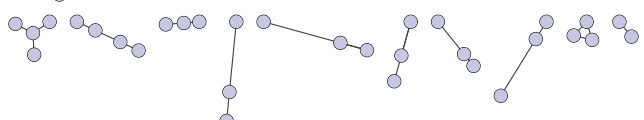

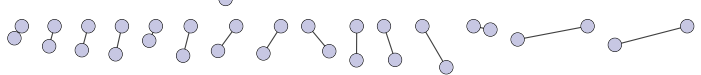

a

$+0$
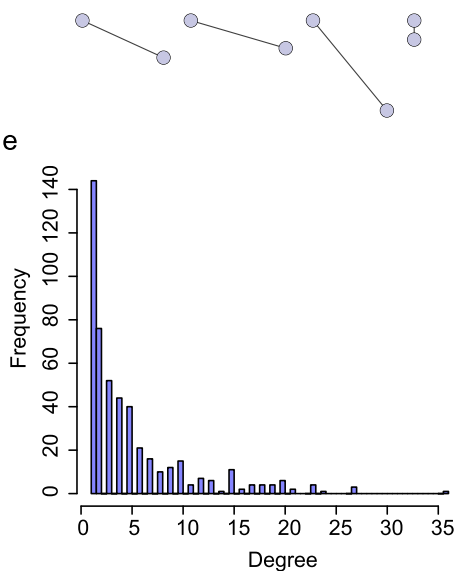

b
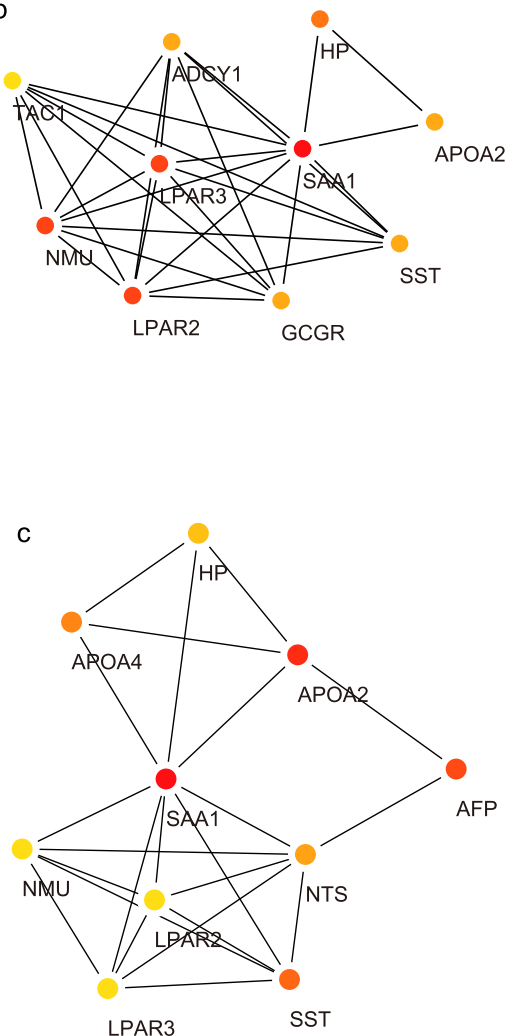

d

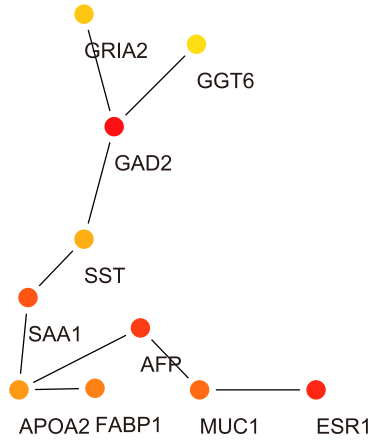

g

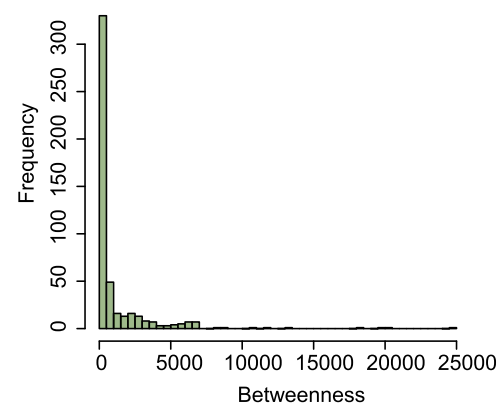

Fig. 2 Screening of hub genes involved in the development of STAD. a The network showed co-expression gene in PPI pairs with a score higher than 0.7; b-d Top hub genes identified by the Degree (b), Closeness (c), and Betweenness (d); e- $\mathbf{g}$ The topological properties of the PPI network and the distributions of degree $(\mathbf{e})$, closeness $(\mathbf{f})$, and betweenness $(\mathbf{g})$ 
Table 3 Univariate Cox regression analysis result of 11 genes in the training set

\begin{tabular}{llllll}
\hline Gene & $\boldsymbol{P}$ value & Hazard ratio & Low $\mathbf{9 5 \% C l}$ & High $\mathbf{9 5 \% C l}$ & Coefficient \\
\hline CDX2 & 0.006 & 1.213 & 1.056 & 1.394 & 0.193 \\
PON1 & 0.000 & 0.657 & 0.534 & 0.807 & -0.421 \\
ADH4 & 0.004 & 0.709 & 0.562 & 0.896 & -0.343 \\
RBP2 & 0.008 & 1.277 & 1.066 & 1.529 & 0.244 \\
LCAT & 0.001 & 0.634 & 0.479 & 0.839 & -0.456 \\
GAL & 0.006 & 1.353 & 1.090 & 1.679 & 0.302 \\
LPA & 0.008 & 0.687 & 0.519 & 0.908 & -0.376 \\
CYP19A1 & 0.006 & 1.339 & 1.089 & 1.648 & 0.292 \\
GAST & 0.010 & 1.290 & 1.063 & 1.565 & 0.254 \\
SST & 0.002 & 1.430 & 1.140 & 1.792 & 0.357 \\
UGT1A8 & 0.010 & 1.188 & 1.043 & 1.353 & 0.172 \\
\hline
\end{tabular}

in addition to pT stage $(P<0.001)$ and $\mathrm{pN}$ stage $(P=$ $0.005)$ were independent prognostic indicators of OS (Fig. 6b). In the ICGC HCC dataset, univariate and multivariate analysis of survival revealed that both the 11-gene signature and pTNM stage were independent prognostic indicators of OS (Fig. 6c-d). Overall, these results suggest that the 11-gene signature is a potential independent prognostic factor for HCC.

\section{Construction of a comprehensive nomogram for survival prediction of HCC patients}

In order to maximize the utility of the signature, we further integrated risk score and other prognostic clinical factors identified by univariate Cox regression analysis (Fig. 6b) to construct a novel nomogram for the survival prediction of HCC patients (Fig. 7a). The one-year, three-year and five-year calibration curves of the nomogram verified the consistency between predicted and actual survival probability of HCC patients (Fig. 7b). And also, in the ICGC HCC dataset, the one-year, three-year and five-year calibration curves of the nomogram the nomogram showed a similar result (Fig. 7c-d).

\section{GSEA analysis of enriched pathway based on risk score} ssGSEA was performed to determine the potential related pathways according to patients' prognostic risk in TCGA, ICGC and GSE15654 cohorts, and pathways with False Discovery Rates (FDR) $<0.05$ were derived. By divided samples into high-risk group and low-risk group based on whether the Riskscore is greater than 0 , and analyzed the enriched pathway in both groups by using GSEA, we found that a total of 20 pathways were identified in the TCGA HCC cohort (Supplementary Table S6), 15 pathways were identified in the ICGC HCC cohort (Supplementary Table S7), and 46 pathways were identified in the GSE15654 cohort (Supplementary Table S8). As top pathways showed in Supplementary Figure 4, all of them were significantly enriched in the low-risk group. Thus, the 11-gene signature may involve in the development and progression of $\mathrm{HCC}$ by participating these pathways.

\section{Discussion}

Since the theory of cancer stem cells (CSCs) was proposed, CSCs have been well recognized and characterized in many human malignancies including hepatic cancer [3]. Cumulative evidences have been yielded in the contribution of CSCs on the development of cancer recurrence, metastasis, and chemo- and radio-resistance in hepatocellular carcinoma [9]. However, several physiopathology and mechanistic questions of hepatic CSC still need to be illuminated. In this study, we identified the molecular subtypes of HCC based on the expression of stemness-related genes (SRGs), which provided a new molecular subtype classification of HCC, and further studied the genomic background of the molecular characteristics of HCC. In addition, we constructed a PPI network based on the 987 differential expression genes obtained from DEseq analysis of the differential genes in each subtype; then constructed a 11-gene signature prognostic model based on the hub genes in the PPI network; after a three-phase training, test and validation process, we confirmed that the 11-gene signature is able to exert stable prediction performance in datasets from different platforms, which means that it has strong robustness on classification of the prognostic risk of patients with HCC.

The associations between SRG expression and immune-infiltration or clinical outcomes have been detected before but has not been explored in HCC [1012]. In our analysis, based on the expression profile of prognostic SRGs, we demonstrated that the Cluster 2 subtype is associated with the highest infiltration of immune cells, and we identified that tumors with more active SRG expression have higher immune-infiltration in tumor microenvironment (TME) and significantly worse 

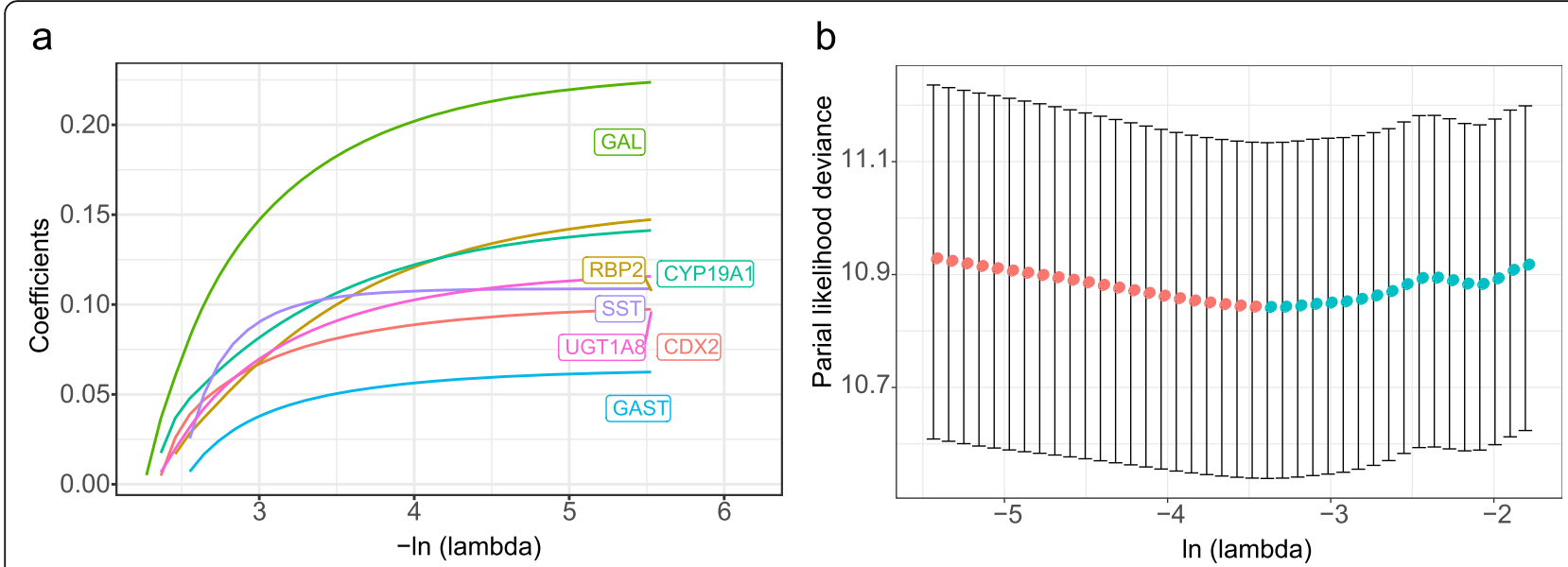

C

d
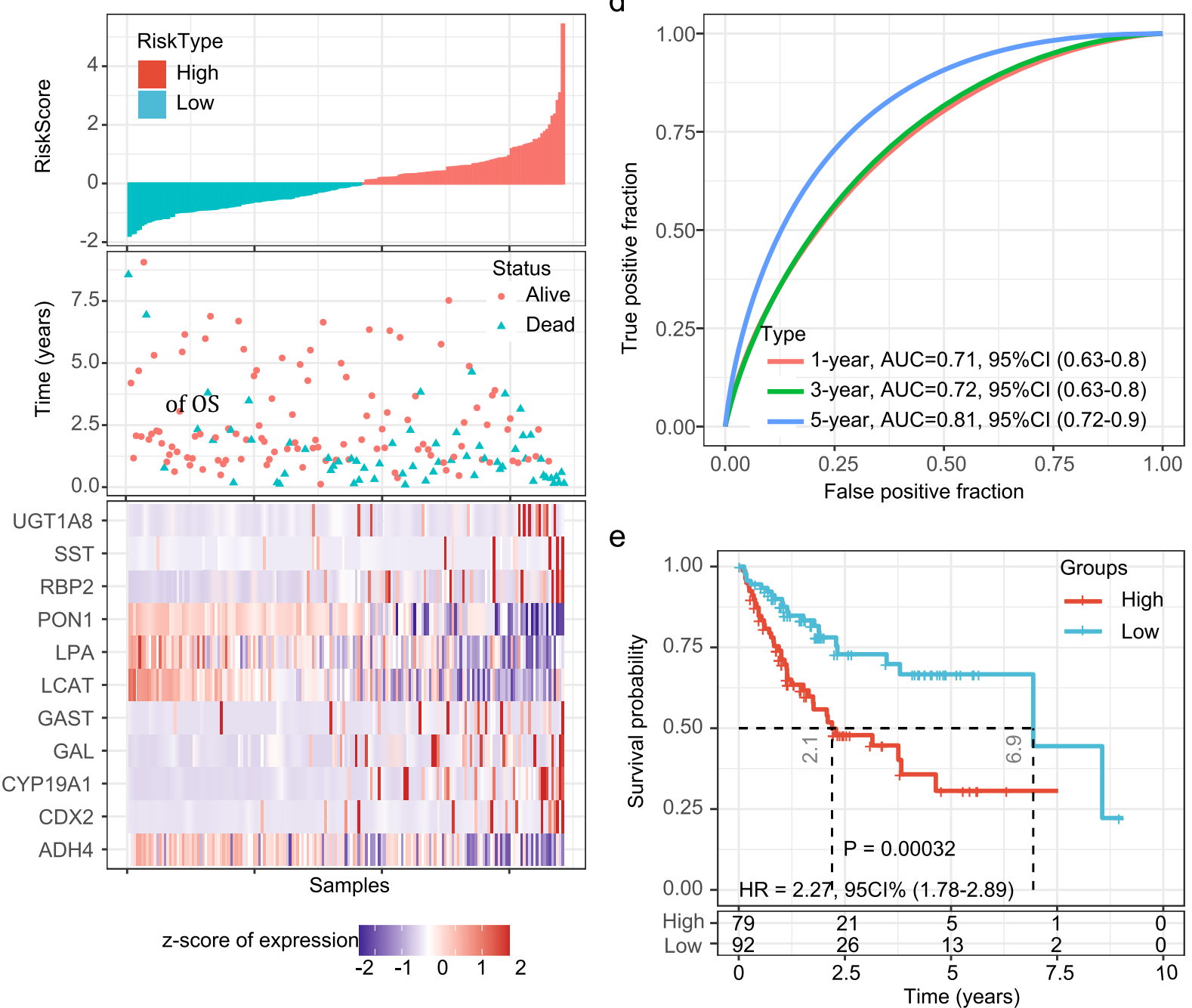

Fig. 3 Evaluation of the performance of the 11-gene signature in the training dataset. a Trajectory change of each independent variable, the $X$ axis represents the log value of the independent variable lambda, the $Y$ axis represents the coefficient of the independent variable; $\mathbf{b}$ Confidence intervals of each lambda; c Risk score, survival time, survival status and expression of the 11-gene signature in the training set. $\mathbf{d}$ ROC curve of the 11-gene signature for OS in the training set. e Kaplan-Meier survival analysis of OS for patients in the training set. AUC, area under the curve; HR, hazard ratio; $\mathrm{Cl}$, confidence interval 

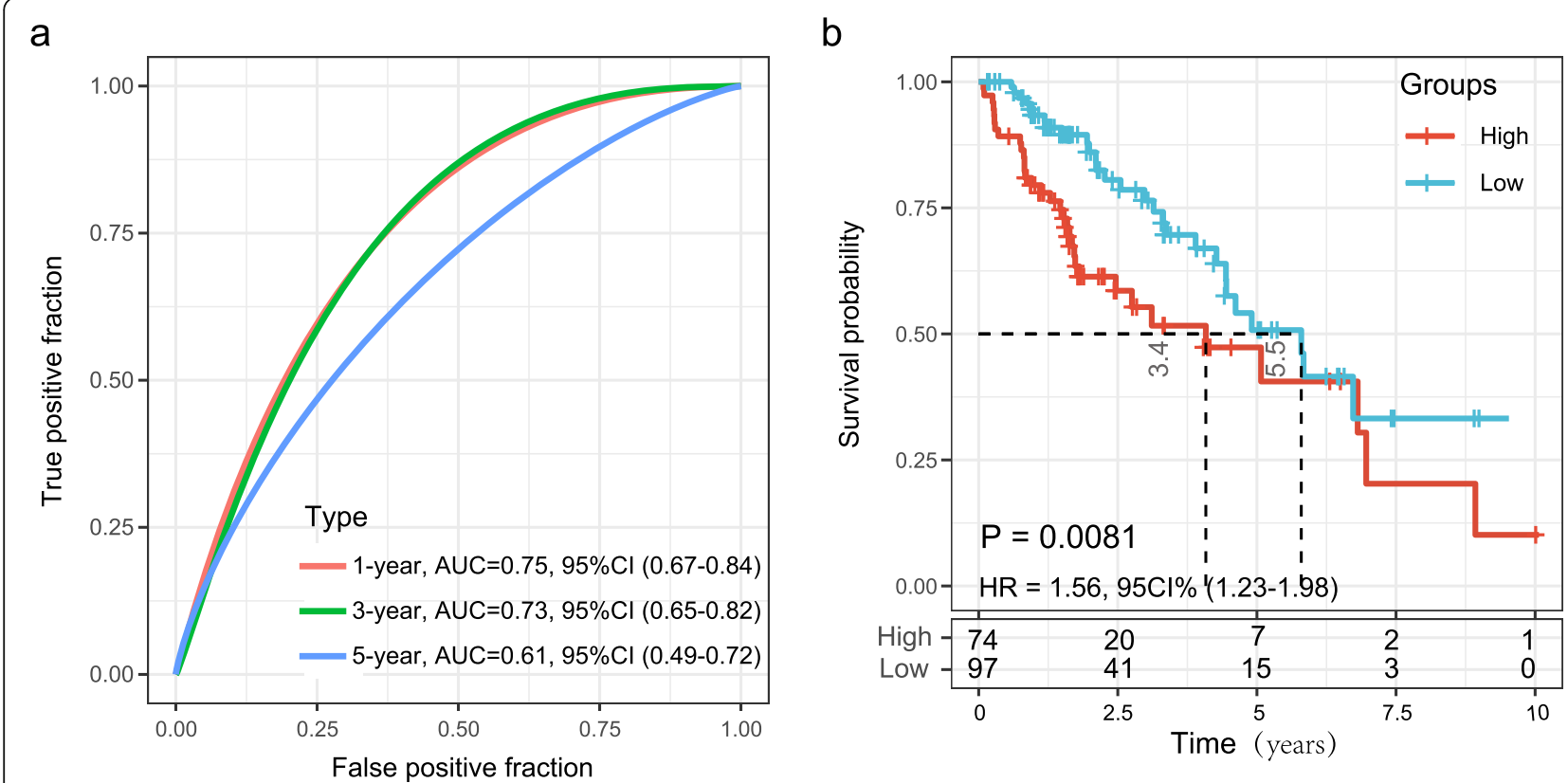

C

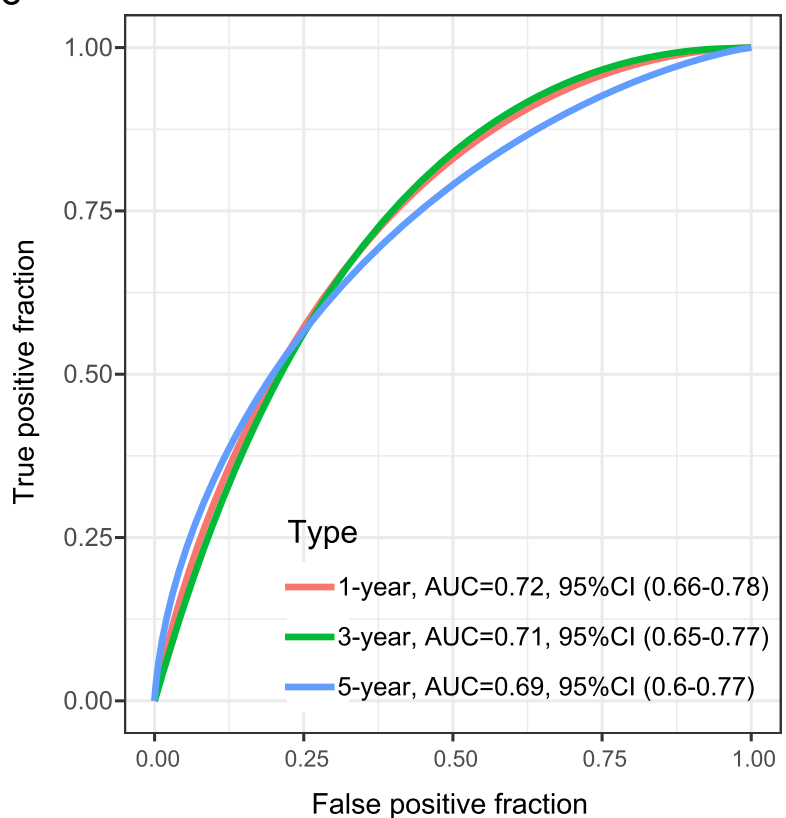

d

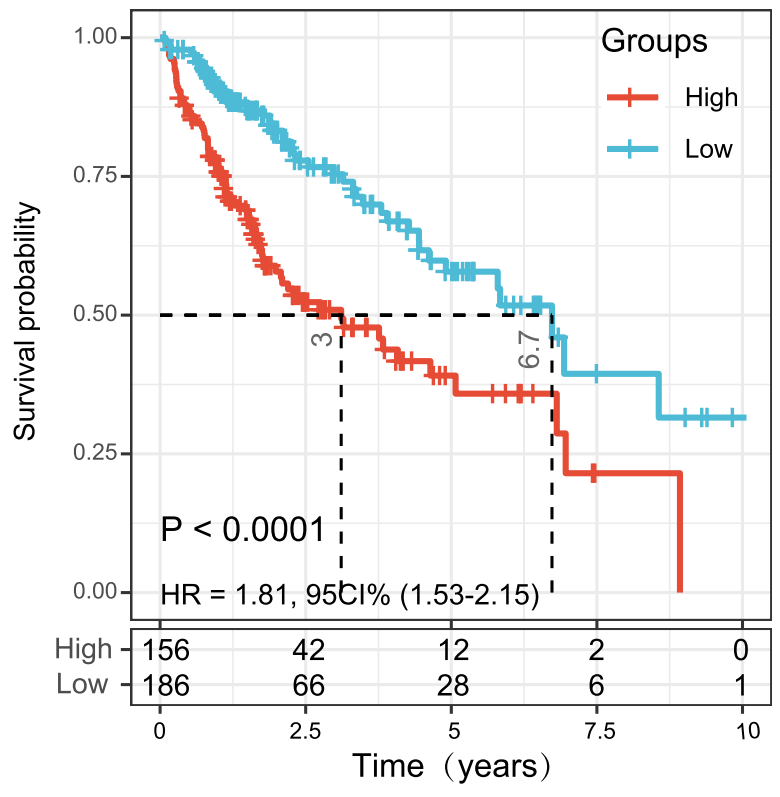

Fig. 4 Internal validation of the 11-gene signature's robustness in the TCGA validation cohort and the entire TCGA cohort. a ROC curve of the 11gene signature for OS in the TCGA validation set; $\mathbf{b}$ Kaplan-Meier survival analysis of OS for patients in the TCGA validation set; $\mathbf{c}$ ROC curve of the 11-gene signature for OS in the entire TCGA set; $\mathbf{d}$ Kaplan-Meier survival analysis of OS for patients in the entire TCGA set

prognosis, which means that increased expression of stemness-related genes and increased immune infiltration may contribute to a poor prognosis. CSCs can show special characteristics to evade the recognition of innate and adaptive immunity, transform TME into immunosuppressive and promote tumorigenic landscape. In addition, immune cells sculpted by CSCs can affect the differentiation and phenotype of tumor cells in TME [13]. Therefore, tumors with more active SRG expression may have higher immune-infiltration in the tumor microenvironment and significantly worse prognosis. Thus, CSCs maintain the malignant phenotype of tumor cells and induce poor clinical outcomes by remodeling the immune contexture. Various stemnessrelated pathways such as Notch and Hedghog signaling are also widely involved in tumor immune regulation; in addition, CSCs can induce immune escape by activating their own oncogenic pathways, such as Wnt and Hippo 

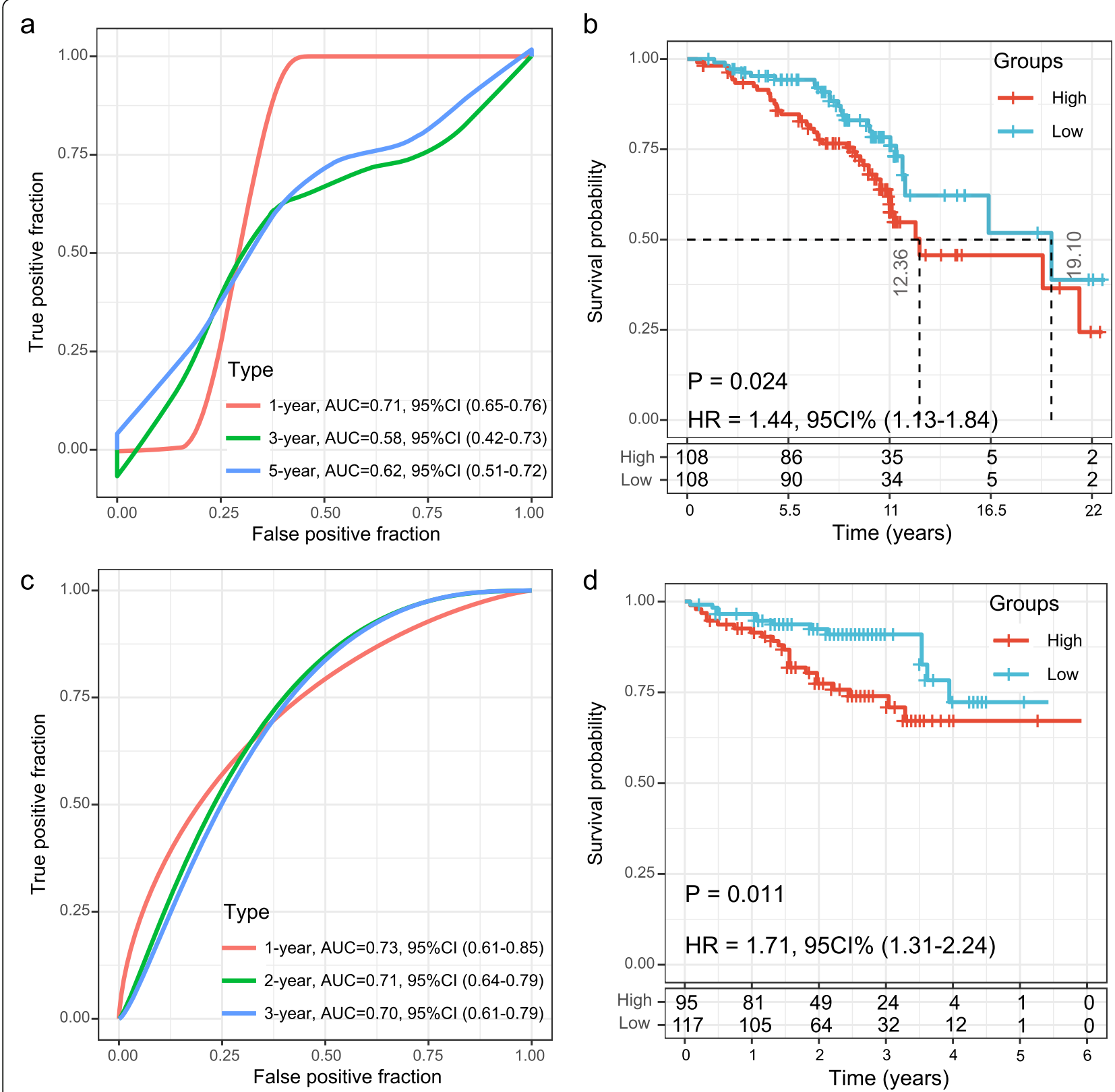

Fig. 5 External validation of the 11-gene signature's robustness in the GSE15654 and ICGC HCC cohorts. a ROC curve of the 11-gene signature for OS in the GSE15654 cohort; b Kaplan-Meier survival curve of OS for patients with HCC based on the 11-gene signature in the GSE15654 cohorts. c ROC curve of the 11-gene signature for OS in the ICGC HCC cohort; $\mathbf{d}$ Kaplan-Meier survival curve of OS based on the 11-gene signature in the ICGC HCC cohorts

signaling, etc. [14]. The expression activity of SRGs to some extent represent the immunomodulatory properties of CSCs; in addition, this immune-related difference in SRGs based molecular subtype may reflect the effects of stemness on TME and the reason why a part of HCC is so fatal regardless of aggressive therapy. Targeting SRGs will facilitate the development of current therapeutic modalities and the R\&D of ground-breaking strategies. Our study suggests a great potential for the use of
SRG profiling as a powerful marker in prognostication and inform treatment decisions for HCC patients.

We further established a 11-gene signature that could classify patients' overall survival. Among the 11 biomarker genes (CDX2, PON1, ADH4, RBP2, LCAT, GAL, LPA, CYP19A1, GAST, SST and UGT1A8) discovered by the present study, caudal-related homeobox 2 (CDX2) is an intestinal-specific homeobox transcription factor that plays a crucial role in the development, 


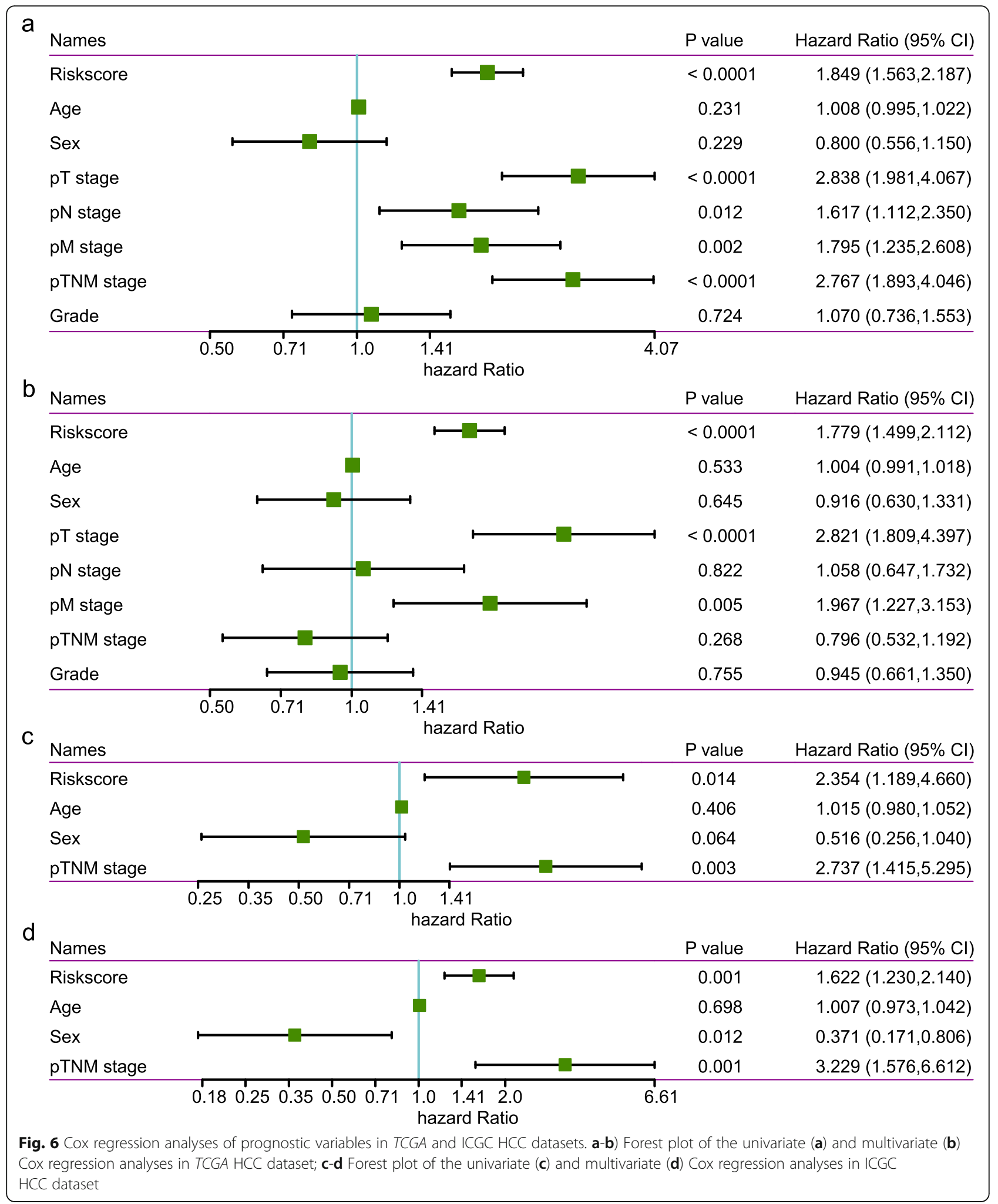

proliferation, and differentiation of intestinal epithelium $[15,16]$. CDX2 have emerged as important modulators of cancer aggressiveness and can influence the viability of HCC cells by transcription regulating oncogene CDH17 [17]. Moreover, CDX2 also implicated in the differentiation of human and mouse pluripotent stem cells 


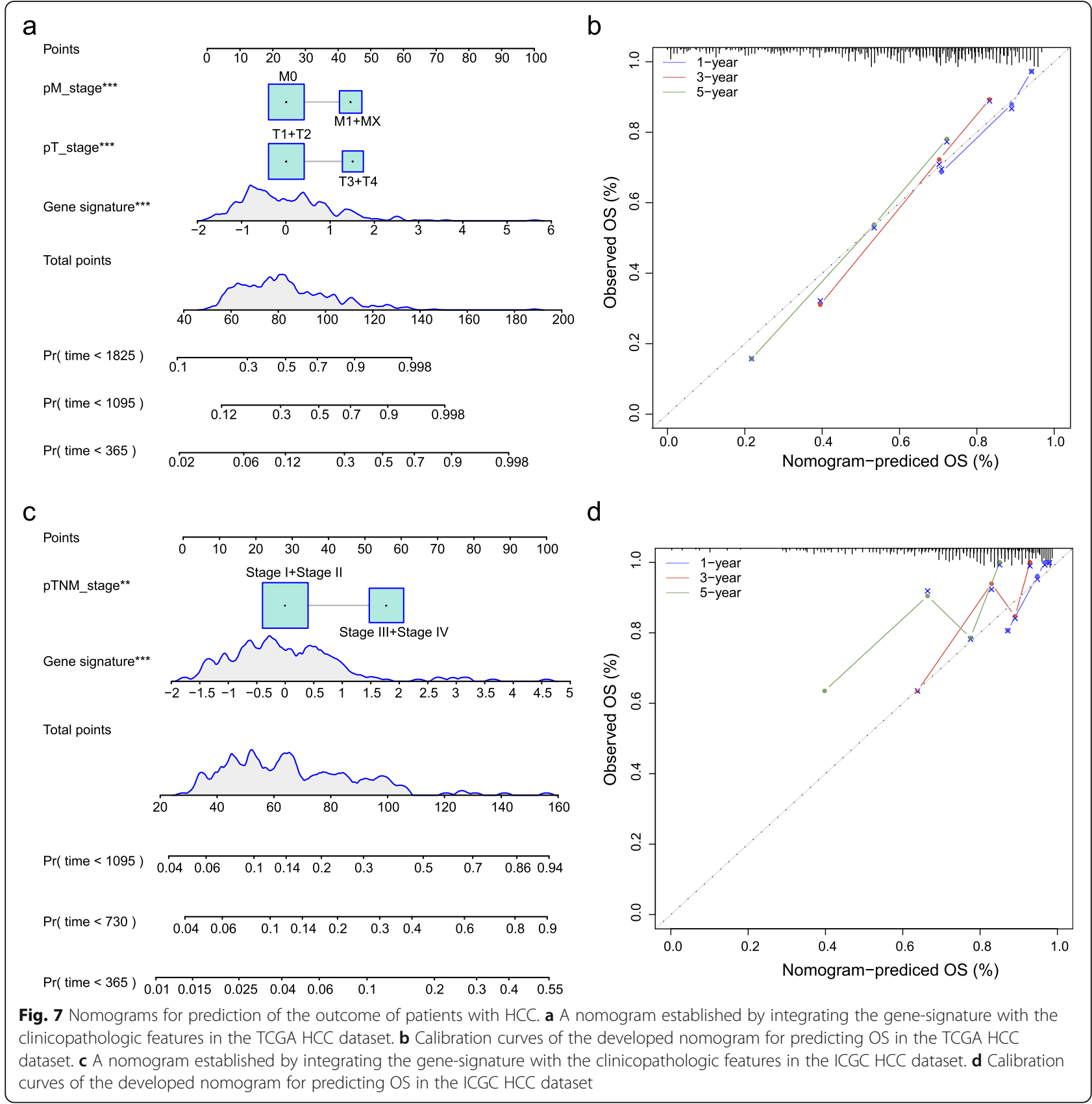

[18]. Retinoblastoma-Binding Protein 2 (RBP2) is a histone demethylase over-expressed in HCC [19], and RBP2 can induce CSC phenotypes through converting renal cell carcinoma cells into a more mesenchymal phenotype [20]. The promoter gene polymorphism of CYP19A1 has been linked with the risk of hepatocellular carcinoma [21, 22]; in addition, it can promote the metastatic homing and proliferation of stem-like prostate cancer cells in the bone marrow [23]. The question that whether CDX2, RBP2, and CYP19A1 involve in the behavior regulation of CSCs subgroup in HCC should be further recognized. Gastrin (GAST) is a trophic factor within the normal gastrointestinal tract and its precursor forms can express in HCC [24]. Interestingly, GAST can also expresses in a group of primary human tumors, including neuronal, renal, and myogenic stem cell tumors [25], which suggest that GAST may play a previously unrecognized role in human CSC. Galanin and GMAP Prepropeptide (GAL) has been demonstrated to activate in human HCC, and it prefers to accumulate in the stromal tissue around the HCC cells [26]; Somatostatin (SST) is a kind of hormone that can inhibits the release 
of various secondary hormones; Paraoxonase 1 (PON1) was found to be a highly related predictor of recurrence and metastasis in HCC [27]; Production and Homeostasis of Lipoprotein(A) (LPA) can be impaired when liver cancer occurs [28]; and both the downregulation of PON1 [27] and alcohol dehydrogenase 4 (Class II), Pi polypeptide (ADH4) [29] can confers poor survival time of HCC. However, the relationship among these genes and CSC or stemness was seldom reported. Besides, seldom has been recognized on the expression or role of Lecithin-Cholesterol Acyltransferase (LACT) and UDP Glucuronosyltransferase Family 1 Member A8 (UGT1A8) in HCC or even CSCs. Nevertheless, although some of previous studies have identified some these genes as prognostic marker in HCC, whereas they were limited by just single gene detected, small cohort, and deficiency on independent validation. The use of the LASSO Cox regression model [30] and nomogram [31] allowed us to integrate multiple genes into one module, which has significantly higher prognostication performance than that of single gene alone or even some previous reported gene signatures.

Some limitations of this study should be taken into consideration. Firstly, since the TCGA, ICGC and GSE15654 datasets we enrolled only included Caucasian population, most of which are hepatitis C-related HCC, this present study may not include patients with hepatitis B-related HCC from other areas loading distinct genetic phenotypes and clinical characteristics, making it vulnerable to the intrinsic biases of such a research format. Obviously, our results should be training in hepatitis B-related HCC cohorts, and further validated by prospective study in some worldwide multicenter clinical study. Moreover, its area under ROC curve should be optimized to improve the prognostic accuracy, and also its prediction value in early HCC must be further evaluated. In addition, despite growing studies began focus on the interaction of tumor cells and associated stemness in human malignancies, most SRG are not yet functionally annotated in HCC, and the biofunction of our 11 genes have not yet been fully investigated in previous studies. Although the physiopathological roles of the gene signature were annotated using computational approaches, additional studies should be carried out to further disclose the mechanisms of these 11 genes involved in the carcinogenesis of HCC. Further, more evidences are required to find out the biological foundation of their dysregulation in $\mathrm{HCC}$.

\section{Conclusions}

In summary, for the first time, we profiled the stemness related molecular subtype in HCC and our study may provide an assessment approach for the CSC-based classification of HCC. Moreover, we identified a stemness-related gene signature which could classify the prognostic risk of patients with HCC. This method might, therefore, help with patient management and individualized therapy of patients with HCC.

\section{Abbreviations}

ADH4: Alcohol dehydrogenase 4 (Class II), Pi polypeptide; CDF: Cumulative distribution function; CDX2: Caudal-related homeobox 2; CSC: Cancer stem cell; DCA: Decision curve analysis; DEG: Differentially expressed gene; DFS: Disease specific survival; EMRG: Energy metabolism-related genes; FDR: False discovery rate; FPKM: Fragments Per Kilobase of exon model per Million mapped fragments; GAL: Galanin and GMAP Prepropeptide; GAST: Gastrin; GEO: Gene Expression Omnibus; HCC: Hepatocellular carcinoma; ICGC: International Cancer Genome Consortium; KEGG: Kyoto Encyclopedia of Genes and Genomes; LACT: Lecithin-Cholesterol Acyltransferase; LASSO: Least absolute shrinkage and selection operator; MSigDB: Molecular Signature Database; OS: Overall survival;

PAC: Ambiguously clustered pairs; PCA: Principal component analysis; PON1: Paraoxonase 1; PPI: Protein-protein interaction; RBP2: RetinoblastomaBinding Protein 2; ROC: Receiver operating characteristic; SRG: Stemness related gene; sSGSEA: Single-sample Gene set enrichment analysis; STRI NG: Search Tool for the Retrieval of Interacting Genes; TCGA: The Cancer Genome Atlas; TME: Tumor microenvironment; UGT1A8: UDP

Glucuronosyltransferase Family 1 Member A8

\section{Supplementary Information}

The online version contains supplementary material available at https://doi. org/10.1186/s12885-021-08351-0.

\begin{abstract}
Additional file 1: Supplementary Figure 1. Distribution of clinicopathological parameters in the four subtypes. Supplementary Figure 2. (A-D) Volcano map of differentially expressed genes (DEG) between each subtype and the other subtypes; (E-F) The Venn diagrams of the overlapping genes among the DEGs in each molecular subtype. Supplementary Figure 3. Distribution of clinicopathological parameters including vascular invasion (A) and tumor differentiation (B) between the high-risk and low-risk groups. Supplementary Figure 4. SSGSEA result according to the risk-score of HCC samples in each dataset, enrichment pathways that were significantly correlated in the low-risk groups (FDR < 0.05). Supplementary Table 1. Pathways related to cancer stem cells in Reactome and GO databases. Supplementary Table S2. List of SRGS correlated with the overall survival of patients with HCC. Supplementary Table S3. Protein-protein interaction pairs with a score higher than 0.7. Supplementary Figure S4. Hub genes identified by the Degree, Closeness and Betweenness. Supplementary Table S4. Betweenness in the PPI network. Supplementary Figure S5. Genes with high degree, closeness, and betweenness scores. Supplementary Table S6. Pathways identified in the TCGA HCC cohort. Supplementary Table S7. Pathways identified in the ICGC HCC cohort. Supplementary Table S8. Pathways identified in the GSE15654 cohort.
\end{abstract}

\section{Acknowledgments}

The authors would like to thank all researchers contributed to the TCGA, ICGC and GEO data sets included.

\section{Authors' contributions}

$\mathrm{HL}$ and $\mathrm{ZS}$ designed the study and revised the manuscript. LH and YZ conducted the data process, modal establishment and visualization of analysis. ZS, XX, WW and CS did the data analysis and interpretation. HL performed statistical analysis performed data analysis and wrote the manuscript. All authors have read and approved the final manuscript.

\section{Funding}

This study was supported by the Basic Public Welfare Research Project of Zhejiang Province (LY19H60015); Zhejiang Medical and health science Technology Project (2019KY205, 2020KY927). These funding bodies played no role in the design of the study and collection, analysis, and interpretation of data and in writing the manuscript. 


\section{Availability of data and materials}

The datasets generated and analyzed during the current study are available in the TCGA repository (https://portal.gdc.cancer.gov/), ICGC database (https://icgcportal.genomics.cn/) and the GEO repository (https://www.ncbi. nlm.nih.gov/geo/).

The datasets generated and analyzed during the current study are available in the TCGA repository (https://portal.gdc.cancer.gov/) with the accession number TCGA-LIHC; the ICGC Data Portal (https://icgcportal.genomics.cn/) with the accession number HCC; and the GEO repository (https://www.ncbi. nIm.nih.gov/geo/) the accession number was GSE15654. Public access to these databases is open. Coherently expressed signatures of human metabolism-related pathways were all download from the Reactome pathway database (https://reactome.org/) and derived by aggregating MSigDB version 7.0 gene sets.

\section{Declarations}

\section{Ethics approval and consent to participate}

Not applicable.

\section{Consent for publication}

Not applicable.

\section{Competing interests}

The authors declare that the research was conducted in the absence of any commercial or financial relationships that could be construed as a potential conflict of interest.

\section{Author details}

'Department of Infectious, The Third Affiliated Hospital of Wenzhou Medical University, Ruian, Zhejiang 325200, People's Republic of China. ${ }^{2}$ Department of Interventional, The Third Affiliated Hospital of Wenzhou Medical University, Ruian, Zhejiang 325200, People's Republic of China. ${ }^{3}$ Department of Respiratory, The Third Affiliated Hospital of Wenzhou Medical University, Ruian, Zhejiang 325200, People's Republic of China.

Received: 17 January 2021 Accepted: 12 May 2021

\section{Published online: 19 June 2021}

\section{References}

1. Siegel RL, Miller KD, Jemal A. Cancer statistics, 2020. CA Cancer J Clin. 2020; 70(1):7-30.

2. Yang JD, Hainaut P, Gores GJ, Amadou A, Plymoth A, Roberts LR. A global view of hepatocellular carcinoma: trends, risk, prevention and management. Nat Rev Gastroenterol Hepatol. 2019;16(10):589-604.

3. Thorgeirsson SS. Stemness and reprogramming in liver cancer. Hepatology. 2016;63(4):1068-70.

4. Najafi M, Farhood B, Mortezaee K. Cancer stem cells (CSCs) in cancer progression and therapy. J Cell Physiol. 2019;234(6):8381-95.

5. Chin $\mathrm{CH}$, Chen SH, Wu HH, Ho CW, Ko MT, Lin CY. cytoHubba: identifying hub objects and sub-networks from complex interactome. BMC Syst Biol. 2014;8(Suppl 4):S11.

6. Hughey JJ, Butte AJ. Robust meta-analysis of gene expression using the elastic net. Nucleic Acids Res. 2015;43(12):e79.

7. Tang Z, Li C, Kang B, Gao G, Li C, Zhang Z. GEPIA: a web server for cancer and normal gene expression profiling and interactive analyses. Nucleic Acids Res. 2017;45(W1):W98-W102.

8. Kerr KF, Brown MD, Zhu K, Janes H. Assessing the clinical impact of risk prediction models with decision curves: guidance for correct interpretation and appropriate use. J Clin Oncol. 2016;34(21):2534-40.

9. Nio K, Yamashita T, Kaneko S. The evolving concept of liver cancer stem cells. Mol Cancer. 2017;16(1):4.

10. Yi L, Huang P, Zou X, Guo L, Gu Y, Wen C, et al. Integrative stemness characteristics associated with prognosis and the immune microenvironment in esophageal cancer. Pharmacol Res. 2020;161:105144.

11. Malta TM, Sokolov A, Gentles AJ, Burzykowski T, Poisson L, Weinstein JN, et al. Machine learning identifies stemness features associated with oncogenic dedifferentiation. Cell. 2018;173(2):338-354.e315.

12. Shats I, Gatza ML, Chang JT, Mori S, Wang J, Rich J, et al. Using a stem cellbased signature to guide therapeutic selection in cancer. Cancer Res. 2011; 71(5):1772-80.
13. Müller L, Tunger A, Plesca I, Wehner R, Temme A, Westphal D, et al. Bidirectional crosstalk between Cancer stem cells and immune cell subsets. Front Immunol. 2020;11:140.

14. Clara JA, Monge C, Yang Y, Takebe N. Targeting signalling pathways and the immune microenvironment of cancer stem cells - a clinical update. Nat Rev Clin Oncol. 2020;17(4):204-32.

15. Silberg DG, Swain GP, Suh ER, Traber PG. Cdx1 and $c d \times 2$ expression during intestinal development. Gastroenterology. 2000;119(4):961-71.

16. Mari L, Milano F, Parikh K, Straub D, Everts V, Hoeben KK, et al. A pSMAD/ CDX2 complex is essential for the intestinalization of epithelial metaplasia. Cell Rep. 2014;7(4):1197-210.

17. Zhu R, Wong KF, Lee NP, Lee KF, Luk JM. HNF1a and CDX2 transcriptional factors bind to cadherin-17 (CDH17) gene promoter and modulate its expression in hepatocellular carcinoma. J Cell Biochem. 2010;111(3):618-26

18. Bernardo AS, Faial T, Gardner L, Niakan KK, Ortmann D, Senner CE, et al. BRACHYURY and CDX2 mediate BMP-induced differentiation of human and mouse pluripotent stem cells into embryonic and extraembryonic lineages. Cell Stem Cell. 2011;9(2):144-55.

19. Wang ZY, Yang J, Liu CK, Shen SQ. High expression of retinoblastomabinding protein 2 (RBP2) in patients with hepatocellular carcinoma and its prognostic significance. Med Sci Monit. 2017;23:2736-44.

20. Zhou D, Kannappan V, Chen X, Li J, Leng X, Zhang J, et al. RBP2 induces stem-like cancer cells by promoting EMT and is a prognostic marker for renal cell carcinoma. Exp Mol Med. 2016;48(6):e238.

21. Yuan X, Zhou G, Zhai Y, Xie W, Cui Y, Cao J, et al. Lack of association between the functional polymorphisms in the estrogen-metabolizing genes and risk for hepatocellular carcinoma. Cancer Epidemiol Biomark Prevent. 2008;17(12):3621-7.

22. Koh WP, Yuan JM, Wang R, Govindarajan S, Oppenheimer R, Zhang ZQ, et al. Aromatase (CYP19) promoter gene polymorphism and risk of nonviral hepatitis-related hepatocellular carcinoma. Cancer. 2011;117(15):3383-92.

23. Miftakhova R, Hedblom A, Semenas J, Robinson B, Simoulis A, Malm J, et al. Cyclin A1 and P450 aromatase promote metastatic homing and growth of stem-like prostate Cancer cells in the bone marrow. Cancer Res. 2016;76(8): 2453-64.

24. Caplin M, Khan K, Savage K, Rode J, Varro A, Michaeli D, et al. Expression and processing of gastrin in hepatocellular carcinoma, fibrolamellar carcinoma and cholangiocarcinoma. J Hepatol. 1999;30(3):519-26.

25. Schaer JC, Reubi JC. High gastrin and cholecystokinin (CCK) gene expression in human neuronal, renal, and myogenic stem cell tumors: comparison with CCK-A and CCK-B receptor contents. J Clin Endocrinol Metab. 1999;84(1): 233-9.

26. Spano D, Russo R, Di Maso V, Rosso N, Terracciano LM, Roncalli M, et al. Galectin-1 and its involvement in hepatocellular carcinoma aggressiveness. Mol Med. 2010;16(3-4):102-15.

27. Yu Z, Ou Q, Chen F, Bi J, Li W, Ma J, et al. Evaluation of the prognostic value of paraoxonase 1 in the recurrence and metastasis of hepatocellular carcinoma and establishment of a liver-specific predictive model of survival. J Transl Med. 2018;16(1):327.

28. Jiang JT, $W u C P, X u N$, Zhang $X G$. Mechanisms and significance of lipoprotein(a) in hepatocellular carcinoma. Hepatobil Pancreatic Dis Int. 2009;8(1):25-8.

29. Wei RR, Zhang MY, Rao HL, Pu HY, Zhang HZ, Wang HY. Identification of $\mathrm{ADH} 4$ as a novel and potential prognostic marker in hepatocellular carcinoma. Med Oncol. 2012;29(4):2737-43.

30. Simon N, Friedman J, Hastie T, Tibshirani R. Regularization paths for Cox's proportional hazards model via coordinate descent. J Stat Softw. 2011;39(5):1-13.

31. Lubsen J, Pool J, van der Does E. A practical device for the application of a diagnostic or prognostic function. Methods Inf Med. 1978;17(2):127-9.

\section{Publisher's Note}

Springer Nature remains neutral with regard to jurisdictional claims in published maps and institutional affiliations. 